\title{
Influence of the dynamic dipolar interaction on the current-induced core switch in vortex pairs
}

\author{
Yaowen Liu, ${ }^{1, *}$ Zhiwei Hou, ${ }^{1}$ Sebastian Gliga, ${ }^{2}$ and Riccardo Hertel ${ }^{2}$ \\ ${ }^{1}$ Department of Physics, Tongji University, Shanghai 200092, People's Republic of China \\ ${ }^{2}$ Institute of Solid State Research IFF-9, Jülich Research Center, D-52425 Jülich, Germany
}

(Received 26 December 2008; revised manuscript received 11 February 2009; published 30 March 2009)

\begin{abstract}
The effective manipulation of the orientation of a vortex core by spin-polarized currents introduces the technologically relevant possibility of addressing individual nanomagnets within large arrays. The vortex configuration, which minimizes the interactions between elements, in principle, allows for high integration densities. Using micromagnetic simulations, we find however that the polarity and vorticity of neighboring vortices can strongly modify the parameters necessary to trigger the core switch, increasing, for example, the switching currents as compared to an insolated disk. Such variations are attributed to the formation of charges during the vortex dynamics and can be interpreted using an analytical model based on the generalized Thiele equation. This model takes into account the dynamic dipolar interaction between neighboring vortices.
\end{abstract}

DOI: 10.1103/PhysRevB.79.104435

PACS number(s): 75.75.+a, 75.60.Jk, 75.40.Gb, 75.40.Mg

Magnetic vortex (MV) has received much attention because of its flux closure configuration which, in principle, is allowed to be a candidate for data storage or logic devices. ${ }^{1,2}$ Such flux closure structure is characterized by an in-plane clockwise $(\mathrm{CW})$ or counterclockwise (CCW) circulating magnetization (defined as the vortex circulation $C$ ) around a 10-20 nm central core magnetized perpendicular to the plane (up- or down-core orientations, defined as the vortex polarity p). ${ }^{3,4}$ Toward the realization of the vortex-based devices, the key concept is the understanding of the fundamental properties of vortex structures. Various behaviors of a single magnetic disk including the magnetic susceptibility, vortex stability, as well as its dynamic excitation by field or current pulses have been intensively investigated, for example, in Ref. 5. When the disks are arranged into chains or arrays, the statically dipolar interactions between vortices are generally negligible, but the strong interactions arising from the dynamic behavior of the magnetization in neighboring elements become significant as the separation distance decreases. Typical variations in the quasistatic properties have been revealed, e.g., the susceptibility increases and the nucleation and annihilation fields decrease due to the magnetostatic interaction. ${ }^{6,7}$ The variation in dynamic properties concerning the eigenfrequency spectra in coupled magnetic disks has been theoretically and experimentally demonstrated. ${ }^{8,9}$

The binary states for the vortex-based devices could be identified by the core polarity, ${ }^{10,11}$ the vortex circulation, ${ }^{12}$ or by the vortex handedness. ${ }^{13}$ Therefore, manipulation of the vortex polarity is one of the most challenging aspects. Reversals in the polarity of vortex core (VC) can be achieved with a traditional scheme using a magnetic field perpendicular to the sample plane; however the required magnetic field is larger than $\sim 2.5 \mathrm{kOe} .{ }^{14}$ Recently, the VC switching has been reported by means of a small amplitude of in-plane resonant magnetic field ${ }^{15}$ or a field pulse. ${ }^{16,17}$ The switching mechanism of VC involves the creation of a vortexantivortex (V-AV) pair with opposite polarity with respect to the original vortex. This is shortly followed by the annihilation of the new antivortex with the initial vortex. ${ }^{15-17}$ Such a VC reversal process can also be triggered by applying an in-plane oscillating ${ }^{18,19}$ or pulsed current ${ }^{20,21}$ in a nanodisk or by applying a perpendicular current in a spin-valve nanopillar. ${ }^{22}$ From a technological point of view, such current-driven VC switching is of great importance because the reliable addressing of a single nanodisk inside dense arrays by currents is easier than that by magnetic fields.

In this paper, the effect of the dipolar interaction which arises during the core switching dynamics will be studied. We find that the vortices can interact strongly when they are displaced from their equilibrium position, thereby limiting the densities. These interactions are magnetostatic in nature and surprisingly depend on the core polarization as well as on the vortex circulation. The critical switching current in a pair of magnetically coupled nanodisks is increased, as compared to an isolated disk. The switching behavior of coupled vortices can also be significantly different from that of a single vortex. These simulation results are qualitatively explained using an analytical model.

As a model system, we consider here a series of disk pairs as illustrated in Fig. 1, each disk $(2 R=150 \mathrm{~nm}$ in diameter and $L=20 \mathrm{~nm}$ in thickness) contains a single vortex. We suppose that the two disks are separated by a distance $d$ along the $x$ axis between the centers of disks. In order to

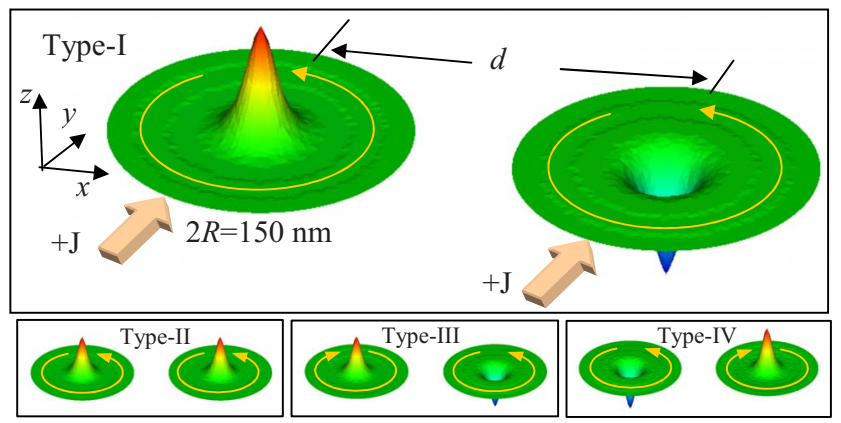

FIG. 1. (Color online) Geometry and topography of the vortex disk pairs with four types of typical initial vortex configurations characterized by their polarity $p$ (up core, +1 ; down core, -1 ) and circulation (counterclockwise $C=+1$; clockwise $C=-1$ ): $\left(p_{1}, C_{1}, p_{2}, C_{2}\right)=(+1,+1,-1,+1),(+1,+1,+1,+1),(+1,-1,-1$, $+1)$, and $(-1,+1,+1,-1)$. Each disk has $150 \mathrm{~nm}$ diameter and 20 $\mathrm{nm}$ thickness. A Gaussian current pulse is applied in the disk plane, parallel to the $y$ axis. 
study the effect of the magnetostatic interaction between the disks, we choose four different unique configurations labeled as "type I," "type II," "type III," and "type IV," respectively, as shown in Fig. 1. The magnetization configurations with the varied combinations of core polarity and vortex circulation correspond to $\left(p_{1}, C_{1} ; p_{2}, C_{2}\right)=(+1,+1,-1,+1)$, $(+1,+1,+1,+1),(+1,-1,-1,+1)$, and $(-1,+1,+1,-1)$, where the subscript $i=1$ is for the left disk and $i=2$ is for the right disk. The reason for choosing the above combinations as the typical configurations of vortex pairs is described as follows. The dynamical trajectory of a VC driven by current has been shown to only depend on the core polarity, showing the CCW rotation trajectory of the core for $p=+1$ and $\mathrm{CW}$ rotation trajectory for $p=-1 .{ }^{18,19}$ Thus, under the actions of a current applied along the $+y$ axis direction, we expect that the two cores for the type I and type III move toward each other but with different magnetic charges of side surface, the cores for the type IV move away from each other, and the cores for the type II move to the same side of the disks.

The magnetization dynamics of magnetically coupled nanodisks are investigated by using a finite-element micromagnetic code based on the Landau-Lifshitz-Gilbert (LLG) equation. $^{23}$ The code has been extended to describe the current-in-plane (CIP) spin-torque effect with two additional terms: ${ }^{20} \quad \mathbf{T}_{a}=-\left(a_{J} / M_{s}^{2}\right) \mathbf{M} \times[\mathbf{M} \times(\partial \mathbf{M} / \partial y)]$ and $\mathbf{T}_{n}=-\left(n_{J} /\right.$ $\left.M_{s}^{2}\right) \mathbf{M} \times(\partial \mathbf{M} / \partial y)$. Here $\mathbf{T}_{a}$ and $\mathbf{T}_{n}$, respectively, denote the adiabatic and nonadiabatic spin-torque terms. ${ }^{24}$ The quantities $a_{J}=P \mu_{B} g j_{e} /\left(2 e M_{s}\right)$ and $n_{J}=\beta a_{J}$, where $P$ is the spin polarization, $\mu_{B}$ is the Bohr magneton, $M_{s}$ is the saturation magnetization, $j_{e}$ is the electric current density, and $\beta$ is the dimensionless constant, describing the degree of the nonadiabaticity. The standard material parameters for Permalloy are used in our simulation: $A=1.3 \times 10^{-11} \mathrm{~J} / \mathrm{m}$ (exchange constant), $\mu_{0} M_{s}=1.0 \mathrm{~T}, \quad K=0$ (anisotropy constant), $\alpha$ $=0.01$ (Gilbert damping constant), $P=0.7$, and $\beta=0.02$. Throughout this paper single Gaussian-shaped current pulses are applied along the $+y$ direction, with a fixed FWHM of 235 ps (i.e., $\sigma=100 \mathrm{ps}$ ) but of varying strength.

The switching behavior of the vortex cores for each configuration is summarized in Figs. 2(a)-2(d) as a function of current pulse strength and interdisk separation. Here we choose three separations $(d=200,250$, and $350 \mathrm{~nm})$ between the paired disks for test. The effective parameter regions for each type of disk pairs which produce a single switch after a current pulse are indicated by the shadows. The simulated threshold and endpoint of current pulse strength are marked by black-solid and red-open scatters, respectively. For comparison, the results for an isolated disk are also indicated in Fig. 2(a). The observed micromagnetic process of the VC reversal for the studied disk pairs is identical to that for an isolated disk described in Refs. 17 and 20. Under the action of a single current pulse, the switching involves a process of creation of a vortex-antivortex pair with opposite polarity with respect to the initial vortex. This is followed by the annihilation of the new antivortex with the initial vortex. ${ }^{17}$ For the 150-nm-diameter isolated disk, similar to our previous result, ${ }^{20}$ the number of times that the core switches depend on the current strength,. A single switch occurs in the range $J=5.8-7.0 \times 10^{12} \mathrm{~A} / \mathrm{m}^{2}$, followed by double switches up to $J=8.0 \times 10^{12} \mathrm{~A} / \mathrm{m}^{2}$. However, simulations for the disk

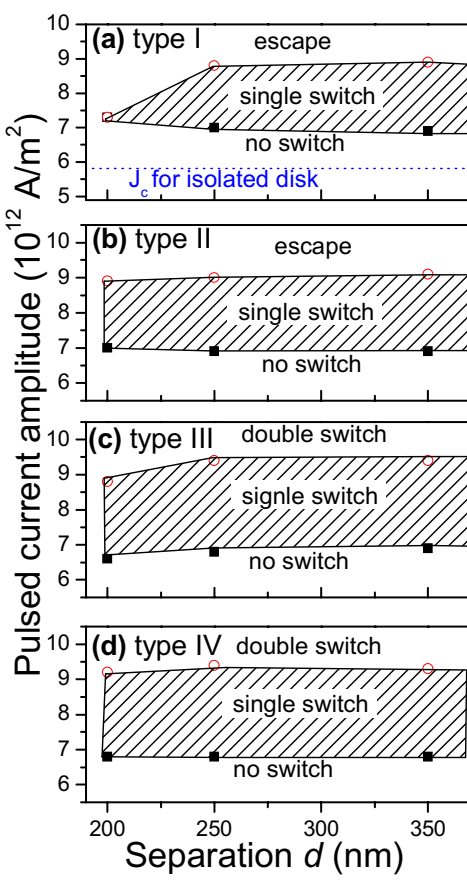

(a') type I, J=7.2, t=580 ps

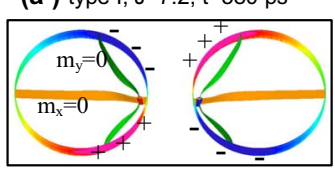

(b') type II, J=7.3, t=440 ps

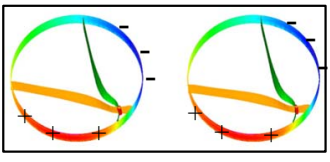

(c') type III, J=7.5, t=420 ps

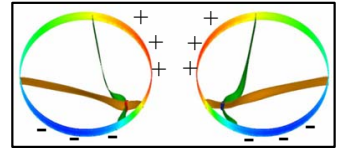

(d') type IV, J=8.3, t=368 ps

FIG. 2. (Color online) Model dependence of the vortex core switching behavior. In the left column, (a)-(d) show the switching current diagrams as a function of the separations $d$ between the paired nanodisks. The full width at half maximum (FWHM) of the current pulse is fixed to 235 ps. The dotted line in the plot of (a) shows the critical switching current for a same-sized isolated disk. In the right column, (a')-(d') show the transient distributions of the side-surface magnetic charges for the different types with $d$ $=200 \mathrm{~nm}$. The applied pulse amplitude (in unit of $10^{12} \mathrm{~A} / \mathrm{m}^{2}$ ) and the moment at which the snapshot was taken are marked in each plot. The color at the disk periphery indicates the polarity and strength change in the surface charges. The brown and green ribbons inside disks represent the $m_{x}=0$ and $m_{y}=0$ isosurfaces, respectively, and the intersection of which determines the exact position of the vortex core.

pairs reveal some significantly different behaviors, as shown in the left panel of Fig. 2. First, the critical switching current is increased for all types of vortex pair configurations. Second, multiple switches of VCs do not occur for the type I and type II by increasing the current strength. Instead, the VCs are expulsed from the sample when the current strength is larger than that of a single switching region. Third, for the very small separation (i.e., $d<200 \mathrm{~nm}$ ) there are even no $\mathrm{VC}$ switches for the type I before the core is expulsed from the disk.

Such significant changes between the disk-pair systems and the isolated disk reveal that the dynamic properties of magnetic vortices are strongly modified when the separation distance is comparable to the diameter. This suggests that the magnetostatic interaction associated with the vortex circulation and core polarity has a strong influence on the parameters, leading to the VC reversal in high-density disk arrays. In order to gain insight of the underlying physics, the Thiele collective coordinate approach ${ }^{25}$ is employed to analytically model the pair of magnetically coupled disks. Taking into account $|\mathbf{M}|=$ const, the LLG equation including adiabatic 
and nonadiabatic spin-torque terms for each disk $(i=1,2)$ can be transformed into two coupled equations with the polar angle $\theta_{i}$ and azimuth angle $\varphi_{i}$,

$$
\begin{gathered}
\frac{\partial \theta_{i}}{\partial t}+\alpha \sin \theta_{i} \frac{\partial \varphi_{i}}{\partial t}=-\frac{\gamma}{M_{s} \sin \theta_{i}} \frac{\partial E_{i}}{\partial \varphi_{i}}+a_{J} \frac{\partial \theta_{i}}{\partial y_{i}}+n_{J} \sin \theta_{i} \frac{\partial \varphi_{i}}{\partial y_{i}} \\
\quad \sin \theta_{i} \frac{\partial \varphi_{i}}{\partial t}-\alpha \frac{\partial \theta_{i}}{\partial t}=\frac{\gamma}{M_{s}} \frac{\partial E_{i}}{\partial \theta_{i}}+a_{J} \sin \theta \frac{\partial \varphi_{i}}{\partial y_{i}}-n_{J} \frac{\partial \theta_{i}}{\partial y_{i}},
\end{gathered}
$$

where $\gamma$ is the gyromagnetic ratio, $\alpha$ is the Gilbert damping parameter, $E_{i}$ is the energy density which involves the interactions between the pair. For a displaced vortex, the force acting on the core can be described by $\vec{F}(\theta, \phi)$ $=-\int[(\partial E / \partial \theta) \nabla \theta+(\partial E / \partial \phi) \nabla \phi] d V$, where the explicit expressions for $\partial E / \partial \varphi$ and $\partial E / \partial \theta$ can be deduced from Eq. (1). Then the generalized Thiele equation for each disk under the action of CIP current can be written as ${ }^{26,27}$

$$
\mathbf{F}_{i}+\mathbf{G}_{i} \times\left(\mathbf{v}_{i}+a_{J} \hat{\mathbf{y}}_{i}\right)+D_{i} \cdot\left(\alpha \mathbf{v}_{i}+n_{J} \hat{\mathbf{y}}_{i}\right)=0,
$$

where $\mathbf{v}_{i}=d \mathbf{X}_{i} / d t$ is the instantaneous velocity of the core and $\mathbf{X}_{i}=\left(X_{i}, Y_{i}\right)$ is the core displacement normalized to the radius of disk. $\mathbf{G}$ is the gyrovector given by $\mathbf{G}=G \hat{z}$ $=-\left(2 \pi \mu_{0} M_{s} L / \gamma\right) p \hat{z}$ and $D$ is the dissipation tensor given by $D=-\left(\pi \mu_{0} M_{s} L / \gamma\right) \ln (R / a)(\hat{x} \hat{x}+\hat{y} \hat{y})$, where $G$ and $D$ are in the same order, the $\hat{x}, \hat{y}, \hat{z}$ are the unit vectors, and $a$ is the lattice constant. ${ }^{27}$ Using the formula of $n_{J}=\beta a_{J}$ and neglecting the higher-order terms, the velocity of vortex core can be approximately derived from Eq. (2),

$$
\begin{gathered}
V_{x} \approx \frac{D(\alpha-\beta)}{G} a_{J}-\frac{\alpha D}{G^{2}} F_{x}-\frac{1}{G} F_{y}, \\
V_{y}=-\frac{G^{2}+\alpha \beta D^{2}}{G^{2}+\alpha^{2} D^{2}} a_{J}+\frac{F_{x} G-F_{y} D \alpha}{G^{2}+\alpha^{2} D^{2}} \approx-a_{J}+\frac{F_{x}}{G}-\frac{D \alpha F_{y}}{G^{2}},
\end{gathered}
$$

where $F_{x}$ and $F_{y}$ are the two components of the force $\mathbf{F}$ acting on the vortex core along the $x$ and $y$ directions, respectively. In the absence of external magnetic field, the $\left(F_{x}, F_{y}\right)$ is provided mainly by the so-called "restoring force" which is generated by the magnetic charges at the disk periphery. $^{28}$

Let us first discuss the initial motion direction of vortex core driven by the current. In this case, the vortex core is located at the center of disk, such initial symmetric vortex configuration leads to a vanishing restoring field, $\mathbf{F}=0$. The initial velocity of vortex core can be evaluated from Eq. (3),

$$
\begin{gathered}
V_{x 0} \approx(\alpha-\beta) \ln (R / a) p a_{J} / 2, \\
V_{y 0} \approx-a_{J} .
\end{gathered}
$$

Equation (4) describes that the initial VC motion direction driven by current is dominated by the vortex polarity $p$ and is independent of the vortex circulation $C$. This is confirmed by micromagnetic simulations performed on an isolated disk with varied vortex configurations shown in Fig. 3. Here the instantaneous core position is exactly identified by the isosurface representations. The cores are located at the intersec-
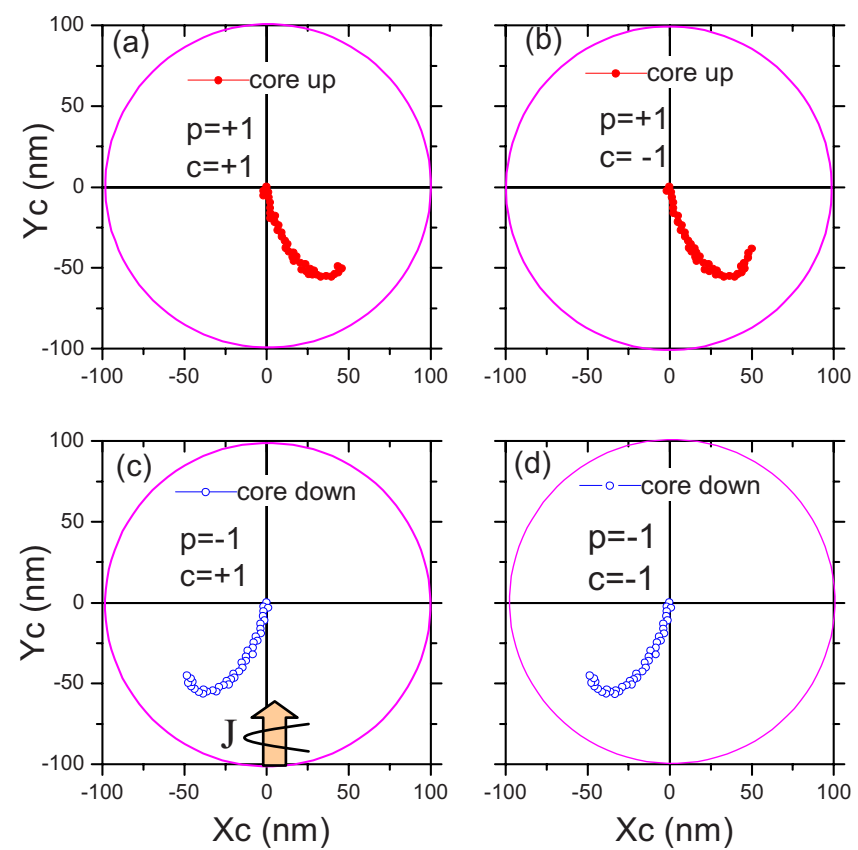

FIG. 3. (Color online) Core trajectories for an isolated disk with various vortex configurations triggered by an in-plane current pulse along the $y$ axis, where the red-solid dots denote the trajectories of the up-orientation cores and the blue-open circles denote those of the down-orientation cores.

tion of two ribbons of $m_{x}=M_{x} / M_{s}=0$ and $\mathrm{m}_{y}=M_{y} / M_{s}{ }^{17} \mathrm{In}$ other words, both the $x$ and $y$ components of magnetization are zero and the $z$ component goes to $100 \%$ at the vortex core. As a result, in the case of disk-pair system shown in Fig. 1, when an in-plane current pulse is applied, each core is displaced from its initial equilibrium position, with the VC with the positive polarity $(p=+1)$ moving toward the bottom-right direction and the core with the negative polarity $(p=-1)$ toward the bottom-left direction, as shown in the right panel of Fig. 2 .

The off-centered vortex breaks the magnetization symmetry and results in the formation of magnetic charges at the periphery of the disks, resulting in the increased internal energy. The instantaneous energy density for each vortex $(i$ $=1,2)$ as a function of the $\mathrm{VC}$ position can be written as ${ }^{8}$

$$
E_{i}\left(\mathbf{X}_{1}, \mathbf{X}_{2}\right)=E(0)+(\kappa / 2)\left|\mathbf{X}_{i}\right|^{2}+E_{\text {int }}\left(\mathbf{X}_{1}, \mathbf{X}_{2}\right),
$$

where the first term is the equilibrium energy for a centered vortex $(\mathbf{X}=0)$ and the second term represents the internal energy of each vortex when it is displaced from equilibrium, which contains the exchange and self-magnetostatic energies. $\kappa$ is the stiffness coefficient which is a function of $R$ and $L .{ }^{28}$ The third term is the long-range interdisk magnetostatic energy due to the side-surface charges $\sigma\left(\varphi_{i}\right)$ generated by the displaced vortices, ${ }^{8}$

$$
\begin{aligned}
E_{\mathrm{int}}\left(\mathbf{X}_{1}, \mathbf{X}_{2}\right) & =\frac{\mu_{0} M_{s}^{2} R^{3}}{8 \pi} \int \frac{d z_{1} d z_{2} d \varphi_{1} d \varphi_{2} \sigma\left(\varphi_{1}\right) \sigma\left(\varphi_{2}\right)}{K\left(\varphi_{1}, \varphi_{2}, z_{1}, z_{2}\right)} \\
& =C_{1} C_{2}\left(\eta_{x} X_{1} X_{2}+\eta_{y} Y_{1} Y_{2}\right)+o\left(\left|\mathbf{X}^{3}\right|\right),
\end{aligned}
$$

where 


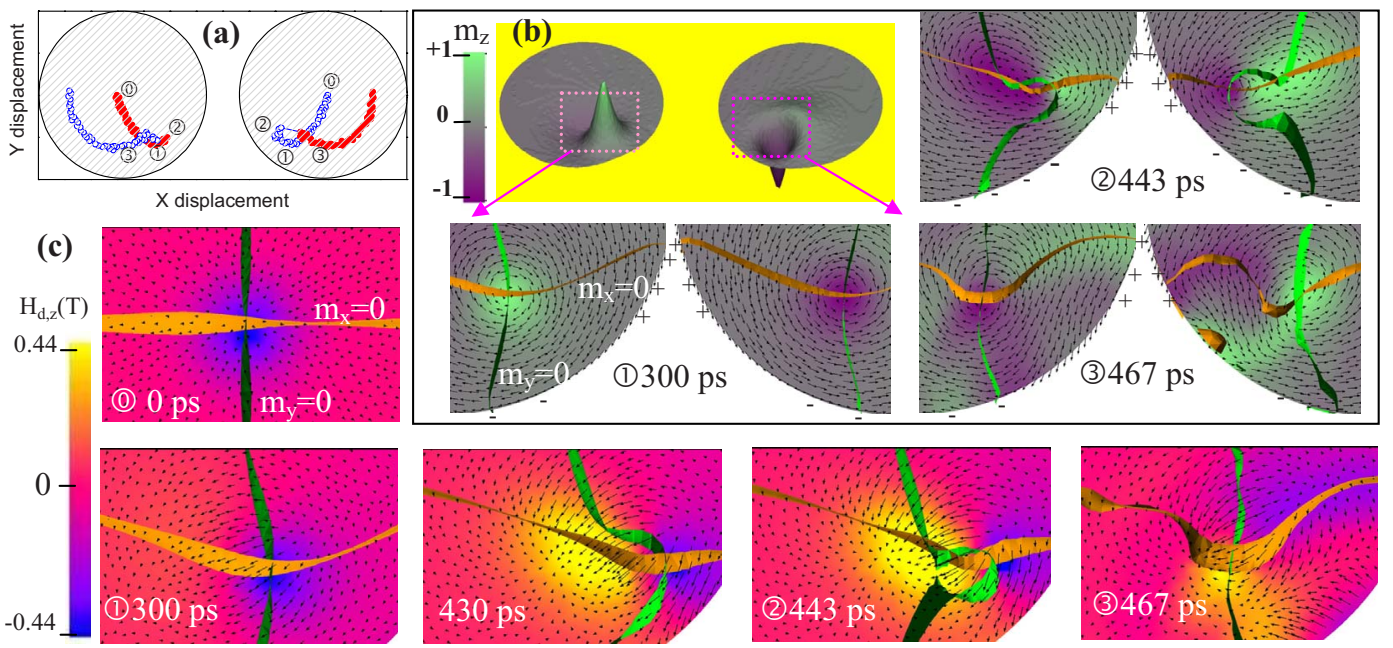

FIG. 4. (Color online) V-AV creation mediated vortex core switching in the case of type-III coupled disk pair with $d=200 \mathrm{~nm}$ driven by a $7.5 \times 10^{12} \mathrm{~A} / \mathrm{m}^{2}$ current pulse. (a) Simulated trajectories of $\mathrm{VC}$ motion. (b) The snapshot images show the spatial $z$ component of magnetization distribution at the indicated times of (a), where the black arrows represent the direction of the in-plane magnetization components. The ribbons that determine the core position are the same as described in Fig. 2. The separation of the disk pair in the images is removed for saving space. (c) Snapshots for the distributions of demagnetizing fields, where the pixels are colored by the perpendicular field component while the in-plane field orientations are given by the arrows.

$$
\begin{aligned}
\sigma\left(\varphi_{i}\right)= & -C_{i}\left(X_{i} \sin \varphi_{i}\right. \\
& \left.-Y_{i} \cos \varphi_{i}\right) / \sqrt{1+\left|\mathbf{X}_{i}\right|^{2}-2\left(X_{i} \cos \varphi_{i}+Y_{i} \sin \varphi_{i}\right)}
\end{aligned}
$$

is the side charge, $K\left(\varphi_{1}, \varphi_{2}, z_{1}, z_{2}\right)=\left[d^{2}+2+2 d\left(\cos \varphi_{2}\right.\right.$ $\left.\left.-\cos \varphi_{1}\right)-2 \cos \left(\varphi_{2}-\varphi_{1}\right)+\left(z_{2}-z_{1}\right)^{2}\right]^{1 / 2}, C_{i}$ is the vortex circu- lation, and $\eta_{x}$ and $\eta_{y}$ correspond to the two integration functions associated to the core position $\mathbf{X}$. It should be noted that the interdisk magnetostatic energy of Eq. (6) ignores the contributions of magnetic charges from the top and bottom surfaces. Inserting Eq. (6) into Eq. (5), we obtain the instantaneous restoring force acting on each vortex core $(i=1,2)$,

$$
\begin{aligned}
F_{1,2} & =\sqrt{\left(F_{x}^{2}+F_{y}^{2}\right)}=\sqrt{\left(\kappa X_{1,2}+C_{1} C_{2} \eta_{x} X_{2,1}\right)^{2}+\left(\kappa Y_{1,2}+C_{1} C_{2} \eta_{y} Y_{2,1}\right)^{2}} \\
& =\sqrt{\kappa^{2}\left(X_{1,2}^{2}+Y_{1,2}^{2}\right)+C_{1}^{2} C_{2}^{2}\left(\eta_{x}^{2} X_{2,1}^{2}+\eta_{y}^{2} Y_{2,1}^{2}\right)+2 \kappa C_{1} C_{2}\left(\eta_{x} X_{1,2} X_{2,1}+\eta_{y} Y_{1,2} Y_{2,1}\right)} .
\end{aligned}
$$

It is worth noticing that the observed increase in the critical switching current in Fig. 2 for the disk pairs can be readily interpreted from the above analytical results. The first term in the square-root expression of Eq. (7) is identical to the restoring force for an isolated vortex disk, ${ }^{5}$ which is independent of the vortex circulation and core polarity. The other two terms are caused by the interactions between the two coupled disks, where the second term gives a positive contribution and the third term actually can be rewritten as $2 k E_{\text {int }}\left(\mathbf{X}_{1}, \mathbf{X}_{2}\right)$ by comparing Eq. (7) with Eq. (5). Therefore, the restoring force acting on the individual disk-pair system is larger than that on an isolated vortex disk. Hence, a larger current is required to displace the vortex and therefore for the core switch to occur in the coupled system.

Moreover, compared with our previous results for the isolated disk, ${ }^{20}$ different switching behaviors are observed in the type I and type II, as shown in Fig. 2; there are no multiple switches when the current strength is increased above the single switch region. But for the type III and type IV, mul- tiple switches occur for large currents. We attribute this variation to the circulation product difference of the vortex pair, e.g., $C_{1} C_{2}=+1$ for the type I and type II but $C_{1} C_{2}$ $=-1$ for the type III and type IV. Equation (7) indicates that the third term for the former two types gives the positive contribution, further increasing the interaction strength while it gives a negative contribution for the latter two types. Consequently, very strong interactions at large current for the types I and II lead to the expulsion of the vortex cores from the disks, as shown in Fig. 2.

To further investigate how the magnetostatic interaction affects the VC reversal process, the vortex dynamics for a type III is shown in Fig. 4. In this case, the left disk has a core orientated "up" and a CW circulation, while the right disk has a "down" core and a CCW circulation. The centerto-center distance between the two disks is $200 \mathrm{~nm}$. When a current pulse with strength of $7.5 \times 10^{12} \mathrm{~A} / \mathrm{m}^{2}$ is applied, the simulated initial trajectories show that the two VCs move toward each other [Fig. 4(a)] [as expected from Eq. (4)]. 
When the off-centered core moves close to the border of disk, magnetic charges appear at the disk circumference [Fig. 4(b)]. The sign of these side-surface charges depends on the vortex circulation in the disk-pair systems, as shown in Figs. 2(a')-2(d'). Such magnetic charges significantly affect the instantaneous energy density of each individual disk [Eqs. (5) and (6)].

Figure 4(c) shows the snapshots of the instantaneous demagnetizing field $H_{d}$ on the left disk, where the color image displays the out-of-plane component of $H_{d}$ and the arrows represent the in-plane components directions; the yellow and green ribbons are the isosurfaces taken at $m_{x}=0$ and $m_{y}=0$, respectively. Note that the dipolar field originated from the neighboring disk is included in $H_{d}$. In the absence of current, the demagnetizing field is strongest in the core region. It points in the $-z$ direction (i.e., opposite to the core magnetization direction) and has no in-plane component [the first image of Fig. 4(c)]. Given the distance between the cores, the magnetic interactions between disks are effectively negligible. When the core is displaced close to the boundary of the disk, magnetic charges are created at the disk circumference. Together with the volume charges from the distorted vortex, they create the in-plane components of $H_{d}$ in the core region. Thus, the local magnetization configuration around the core region was strongly distorted, which in turn further enhances the strength of the demagnetizing field. As a result, the two ribbons at the inner side of the core bend inward. Simultaneously, a dip ( $-z$ direction) is created on the inner side of the core by a possible gyrofield. ${ }^{29}$ When the two ribbons bend enough to form two new intersections, corresponding to the creation of a vortex-antivortex pair, the positive $z$ component of $H_{d}$ is significantly enhanced to $\sim 400 \mathrm{mT}$, see Fig. 4(c). Subsequently, the new vortexantivortex pair undergoes a Kelvin motion to approach the original vortex, then the antivortex annihilates with the original vortex ${ }^{30}$ finally the new vortex with opposite core polarity remains and the core switching process is completed.

In conclusion, we have investigated the dynamic properties of vortex core switching in pairs of magnetically coupled nanodisks driven by an in-plane spin-polarized current. Both micromagnetic simulation and analytical modeling indicate that the dynamic behaviors of the two interacting vortices in a disk-pair system depend on their polarities as well as on the vortex circulations, showing that the larger switching current is required as compared with the insolated disk. We attribute this to the strong interdisk interactions when the vortices are displaced from their equilibrium position. Such variations in dynamic behaviors together with the relative larger dimension would limit the density for the magnetic vortices as the candidate of data storage.

This work was partially supported by the NSF of China (Grant No. 50871075) and Natural Science Foundation of Shanghai (Grant No. 08ZR1420500). Y.L acknowledges Jülich research center for kind hospitality and financial support from the Alexander von Humboldt Foundation.

\footnotetext{
*Author to whom correspondence should be addressed; yaowen@tongji.edu.cn

${ }^{1}$ R. P. Cowburn, J. Magn. Magn. Mater. 242-245, 505 (2002).

${ }^{2}$ S. D. Bader, Rev. Mod. Phys. 78, 1 (2006).

${ }^{3}$ T. Shinjo, T. Okuno, R. Hassdorf, K. Shigeto, and T. Ono, Science 289, 930 (2000).

${ }^{4}$ A. Wachowiak, J. Wiebe, M. Bode, O. Pietzsch, M. Morgenstern, and R. Wiesendanger, Science 298, 577 (2002).

${ }^{5}$ K. Y. Guslienko, B. A. Ivanov, V. Novosad, Y. Otani, H. Shima, and K. Fukamichi, J. Appl. Phys. 91, 8037 (2002); J. P. Park, P. Eames, D. M. Engebretson, J. Berezovsky, and P. A. Crowell, Phys. Rev. B 67, 020403(R) (2003).

${ }^{6}$ K. Y. Guslienko, V. Novosad, Y. Otani, H. Shima, and K. Fukamichi, Phys. Rev. B 65, 024414 (2001).

${ }^{7}$ T. Kimura, Y. Otani, H. Masaki, T. Ishida, R. Antos, and J. Shibata, Appl. Phys. Lett. 90, 132501 (2007).

${ }^{8}$ J. Shibata, K. Shigeto, and Y. Otani, Phys. Rev. B 67, 224404 (2003); J. Shibata and Y. Otani, ibid. 70, 012404 (2004).

${ }^{9}$ G. Gubbiotti, M. Madami, S. Tacchi, G. Carlotti, and T. Okuno, J. Appl. Phys. 99, 08C701 (2006).

${ }^{10}$ R. P. Cowburn, Nature Mater. 6, 255 (2007); J. Thomas, Nat. Nanotechnol. 2, 206 (2007).

${ }^{11}$ S.-K. Kim, K.-S. Lee, Y.-S. Yu, and Y.-S. Choi, Appl. Phys. Lett. 92, 022509 (2008).

${ }^{12}$ K. Bussmann, G. A. Prinz, S.-F. Cheng, and D. Wang, Appl. Phys. Lett. 75, 2476 (1999).

${ }^{13}$ S. Bohlens, B. Krüger, A. Drews, M. Bolte, G. Meier, and
}

D. Pfannkuche, Appl. Phys. Lett. 93, 142508 (2008)

${ }^{14}$ N. Kikuchi, S. Okamoto, O. Kitakami, Y. Shimada, S. G. Kim, Y. Otani, and K. Fukamichi, J. Appl. Phys. 90, 6548 (2001).

${ }^{15}$ B. Van Waeyenberge, A. Puzic, H. Stoll, K. W. Chou, T. Tyliszczak, R. Hertel, M. Fähnle, H. Brückl, K. Rott, G. Reiss, I. Neudecker, D. Weiss, C. H. Back, and G. Schütz, Nature (London) 444, 461 (2006).

${ }^{16}$ Q. F. Xiao, J. Rudge, B. C. Choi, Y. K. Hong, and G. Donohoe, Appl. Phys. Lett. 89, 262507 (2006).

${ }^{17}$ R. Hertel, S. Gliga, C. M. Schneider, and M. Fahnle, Phys. Rev. Lett. 98, 117201 (2007); R. Hertel and C. M. Schneider, ibid. 97, 177202 (2006).

${ }^{18}$ K. Yamada, S. Kasai, Y. Nakatani, K. Kobayashi, H. Kohno, A. Thiaville, and T. Ono, Nature Mater. 6, 270 (2007).

${ }^{19}$ S. K. Kim, Y.-S. Choi, K.-S. Lee, K. Y. Guslienko, and D.-E. Jeong, Appl. Phys. Lett. 91, 082506 (2007).

${ }^{20}$ Y. Liu, S. Gliga, R. Hertel, and C. M. Schneider, Appl. Phys. Lett. 91, 112501 (2007).

${ }^{21}$ K. Yamada, S. Kasai, Y. Nakatani, K. Kobayashi, and T. Ono, Appl. Phys. Lett. 93, 152502 (2008).

${ }^{22}$ Y. Liu, H. He, and Z. Zhang, Appl. Phys. Lett. 91, 242501 (2007).

${ }^{23}$ R. Hertel, W. Wulfhekel, and J. Kirschner, Phys. Rev. Lett. 93, 257202 (2004).

${ }^{24}$ S. Zhang and Z. Li, Phys. Rev. Lett. 93, 127204 (2004).

${ }^{25}$ A. A. Thiele, Phys. Rev. Lett. 30, 230 (1973); D. L. Huber, Phys. Rev. B 26, 3758 (1982). 
${ }^{26}$ A. Thiaville, Y. Nakatani, J. Miltat, and Y. Suzuki, Europhys. Lett. 69, 990 (2005).

${ }^{27}$ J. He, Z. Li, and S. Zhang, Phys. Rev. B 73, 184408 (2006);

B. Krüger, A. Drews, M. Bolte, U. Merkt, D. Pfannkuche, and G. Meier, ibid. 76, 224426 (2007).

${ }^{28}$ K. Y. Guslienko, B. A. Ivanov, V. Novosad, Y. Otani, H. Shima, and K. Fukamichi, Appl. Phys. Lett. 78, 3848 (2001).

${ }^{29}$ K. Y. Guslienko, K. S. Lee, and S.-K. Kim, Phys. Rev. Lett. 100, 027203 (2008).

${ }^{30}$ S. Komineas, Phys. Rev. Lett. 99, 117202 (2007); S. Komineas and N. Papanicolaou, New J. Phys. 10, 043021 (2008). 